
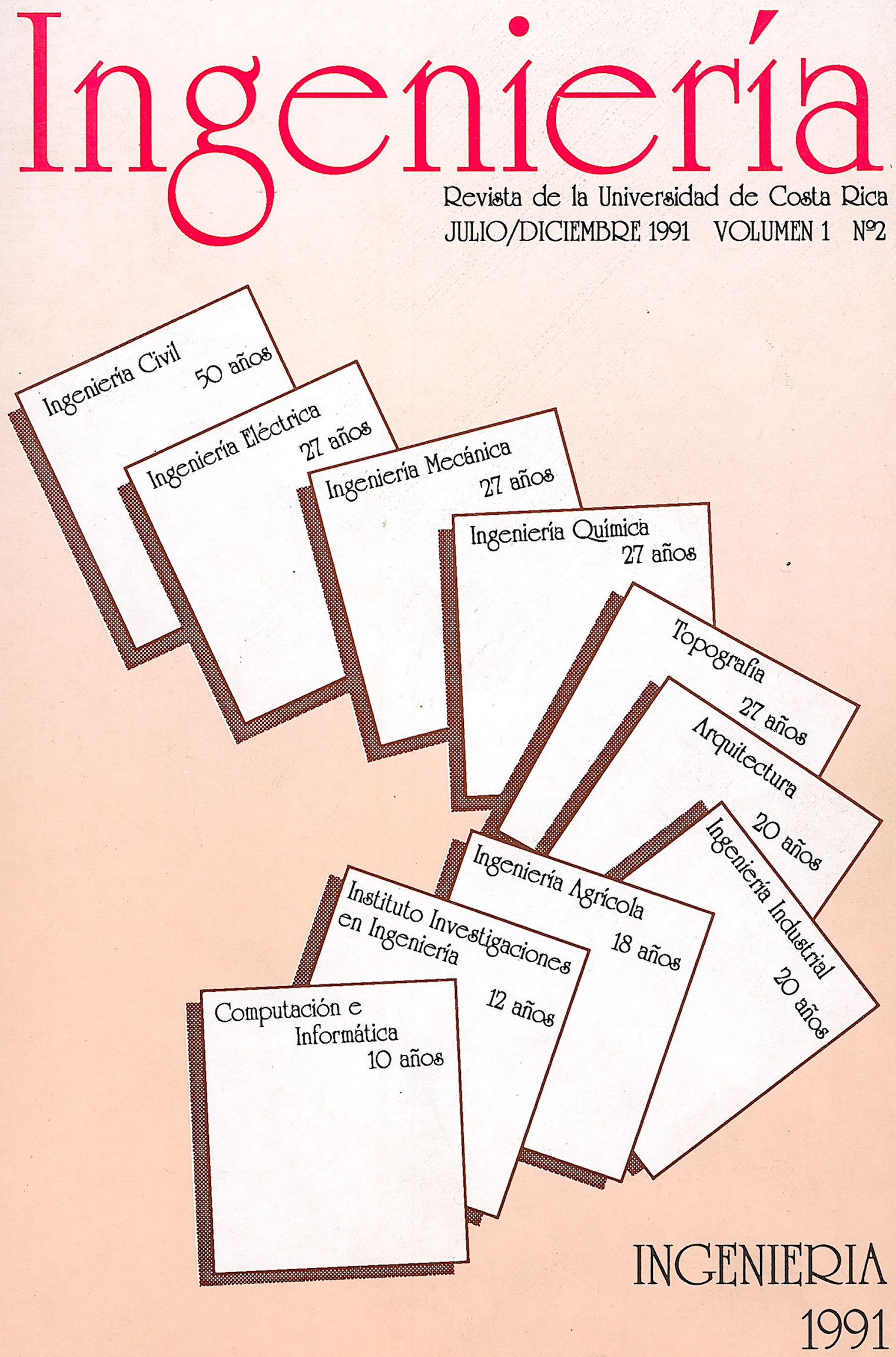


\title{
DESAROLLO DE REDES DE CONMUTACION DE PAQUETES Y OPORTUNIDADES PARA LA UNIVERSIDAD DE COSTA RICA
}

Ing. Eduardo A. Navas, MSc*

\begin{abstract}
Resumen
Este artículo pretende dar una introducción al desarrollo e historia de las redes privadas de conmutación de paquetes y los beneficios que se pueden lograr para la Universidad de Costa Rica al ser parte de la red Bitnet. Se describe el desarrollo de redes privadas orientadas principalmente a las universidades en el mundo.
\end{abstract}

Summary

This article pretends to give an introduction to the development and history of private packet switching networks and to point out the benefits the University of Costa Rica can have by being part of the Bitnet network. It is given a description of the development of private networks oriented to link universities around the world.

\section{INTRODUCCION}

En el siglo XX, la recolección de información, su procesamiento y su distribución ha sido la clave de la tecnología.

Se han desarrollado diferentes tecnologías, como la radio, redes telefónicas, televisión, transmisiones vía satélite y la industria de la computación debido al desarrollo de la electrónica lo cual ha producido que se "acorten las distancias".

Hay organizaciones que tienen muchos departamentos dispersos en una cierta área geográfica y estos departamentos necesitan intercambiar información entre ellos.

Las redes de computadores se han utilizado para lograr este intercambio de información. Las aplicaciones implementadas se pueden resumir en tres grupos principales:

\section{a) Acceso a bases de datos remotas.}

b) Accesos a programas remotos.

c) Mejores facilidades de comunicaciones, como por ejemplo transferencia de mensajes (correo electrónico), transferencias de fondos entre bancos, transferencia de archivos, teleconferencias, etc.

\footnotetext{
- Universidad de Costa Rica Escuela de Ingeniería Eléctrica
}

\section{DESARROLLO HACIA LAS REDES DE CONMUTACION DE PAQUETES}

En los años 50 los computadores usaban el sistema de tarjetas perforadas para entrada de datos. La información era procesada como una aplicación a la vez ("batch processing").

En los años 60 los costos de los computadores empiezan a bajar en relación con los costos de mano de obra del personal, por lo que se generaron nuevas aplicaciones. Se inició el procesamiento en línea ("on-line processing") que permitía a las terminales introducir datos a las computadoras.

En los años 70 hubo más cambios, los minicomputadores podían comunicarse con los computadores centrales ("mainframes") y ellos podían estar en diferentes edificios o ciudades ya que el precio relativo de procesamiento versus comunicaciones bajo considerablemente.

Al final de la década aparecieron los microcomputadores y mucha más gente tuvo acceso a recursos computacionales. En los años 80 el costo de los microcomputadores disminuye considerablemente y se empiezan a utilizar satélites para las comunicaciones entre las computadoras.

Existen muchas clases de redes, una clasificación muy importante está basada en la distancia. En la Tabla No.1 se muestra esta clasificación. 


\section{TABLA No.1 \\ CLASIFICACION DE REDES DE COMPUTADORES}

Distancia entre
Procesadores

Menos de $10 \mathrm{~m}$

Entre $10 \mathrm{~m}$ y $3 \mathrm{Km}$

Entre $3 \mathrm{Km}$ y $50 \mathrm{Km}$

Redes de Area

Metropolitana

(MAN)

Más de $50 \mathrm{Km}$

Redes de Area

Ancha (WAN)
Las primeras redes en implementarse fueron las redes de área ancha debido al hecho de que en los años 70 no se tenían microcomputadoras sino más bien minicomputadoras con sus respectivas terminales no inteligentes, que se deseaban conectar a computadores más grandes dentro de una misma ciudad o otras ciudades. Las redes de área local se desarrollaron a partir del auge de las microcomputadoras y las redes de área metropolitana, que están aún desarrollándose, debido al auge de las redes de área local y la cantidad de información generada en estas.

Las redes de datos de área ancha se pueden dividir en redes de conmutación de paquetes y redes de conmutación de circuitos (sistema telefónico).

La red telefónica es la más difundida en el mundo, es decir tiene un número mayor de puntos de acceso que cualquier otra red. Es por este hecho que normalmente cuando se quiere accesar una red de datos se hace a través de la red telefónica. Esto impone ciertas limitaciones a las redes de área ancha ya que el sistema telefónico fue diseñado teniendo en mente la transmisión de voz sólamente.

\section{REDES PUBLICAS Y PRIVADAS}

Se puede hacer otra clasificación de redes que no depende de sus caractarerísticas técnicas sino más bien de sus posibilidades de acceso. Es decir, podemos dividir las redes de área ancha en redes públicas y redes privadas.

Las redes públicas de datos son administradas en la mayoría de los casos por un ente gubernamental. Al igual que en la red telefónica pública, cualquier persona u organización puede solicitar su servicio. En Costa Rica la red RACSAPAC de RACSA es una red de conmtutación de paquetes pública.

Las redes públicas de datos normalmente siguen el modelo de referencia de sistemas abiertos (OSI) que define la arquitectura de una red, es decir, la estructura, los protocolos y las interfaces de los siete niveles de una red.

Los protocolos que han sido muy aceptados del modelo OSI son los que conforman los tres primeros niveles, y son conocidos como el estándar X.25. Este estándar incluye el X.21 para el nivel físico, el HDLC ("High Level Data Link Control") para el nivel de enlace de datos y la parte final del X.25 para el nivel de red. Debido a que la mayoría de las terminales o microcomputadores que necesitan hacer uso de la red no son compatibles con X.25, se ha estandarizado el protocolo X.3, que incluye el X.28 y X.29 para hacerlas compatibles con X.25. El X.25 brinda entonces un método de acceso prácticamente universal para las redes públicas.

Sin embargo, para los niveles superiores el panorama no está tan claro, es decir el protocolo del nivel de transporte de OSI tiene cinco variantes y no se ha hecho universal su uso. Así mismo, los protocolos de sesión y presentación tampoco han sido tan aceptados porque no todas las aplicaciones necesitan de ellos y finalmente, el nivel de aplicación tiene los protocolos FTAM (Administración, Acceso y Transferencia de Archivos), el MOTIS (Sistemas de Intercambio de Textos Orientado a Mensajes), similar al X.400 del CCITT y el VTP (Protocolo de Terminales Virtuales). De igual forma, no han sido los protocolos más utilizados en las redes de computadores.

Por lo tanto, no está claro cuándo se va a universalizar el uso de los protocolos del modelo OSI.

Las redes privadas, están dispersas por todo el mundo, son administradas por entes privados y los 
usuarios deben cumplir con ciertos requisitos para poder ser admitidos a ellas.

\section{ARPANET}

La primera red desarrollada en el mundo fue ARPANET, auspiciada por la Agencia de Proyectos Avanzados de Investigación (ARPA) del Departamento de Defensa de los Estados Unidos. Esta red de conmutación de paquetes se creó por la necesidad de investigar en el campo de comunicaciones de datos y de compartir recursos entre varios centros de computación distribuidos en diferentes ubicaciones geográficas de los Estados Unidos. La investigación empezó a finales de la década de los años 60 .

Uno de los objetivos principales era que la red no tuviera componentes centrales críticos de forma que su disponibilidad fuera mayor. Se decidió que los centros de cómputo no debían de manejar el procesamiento de datos de control de comunicaciones para el funcionamiento de la red. Por lo tanto, se designó un computador especializado, llamado interfaz del procesador de mensajes (IMP), para que los computadores servidores (Hosts) ingresaran a la red, ver figura No.1.

En 1969 se empezó la primera fase de la red que incluía 14 centros de investigación. Para finales de 1970 se comenzó la segunda fase que incluía a 34 centros de investigación. Hoy en día, se tienen varios cientos de computadoras de diferentes universidades y contratistas del Departamento de Defensa de Estados Unidos alrededor del mundo conectadas a esta red. Además, se han conectado otras redes militares de Estados Unidos a ARPANET utilizando satélites.

Originalmente, los IMPs utilizados eran minicomputadores DDP-516 de Honeywell cuya memoria RAM era de $12 \mathrm{~K}$ palabras de 16 bits cada una. Estos IMPs han ido evolucionando y ahora algunos de ellos se pueden configurar para que usuarios de terminales se conecten directamente a ellos en vez de hacer la conexión a través de un Host. Las velocidades de transmisión de IMPs originales eran de $56 \mathrm{Kbps}$, pero ahora algunos IMPs utilizan velocidades de hasta $230 \mathrm{Kbps}$.

A partir de lo que en 1969 fue un experimento con ARPANET, se empezaron a desarrollar otras redes, sin embargo, se puede afirmar que muchos de los conocimientos que se tienen hoy en día sobre redes de computadoras se deben a ARPANET y por eso se utiliza mucha de la terminología de ARPANET en la literatura técnica de redes.
La red ARPANET utiliza el protocolo de comunicaciones TCP/IP, el cual se desarrollo de una forma muy versatil para interconectar diferentes tipos de redes porque existen bastantes interconexiones de redes de area ancha y local en ARPANET. Los servicios que presta esta red son transferencia de archivos, correo electrónico y teleproceso ("remote login") por medio de los protocolos FTP ("file transfer protocol"), SMTP ("simple mail transfer protocol") y Telnet respectivamente.

La red ARPANET obviamente no sigue el modelo de referencia OSI (Open Systems Interconection) porque fue implementada antes de que se discutiera OSI.

\section{UUCP Y USENET}

Conforme se fue desarrollando ARPANET, otras redes privadas en los Estados Unidos se fueron desarrollando también. La necesidad de intercambiar información fue creciendo y la tecnología fue reduciendo los costos de implementación.

Los computadores con sistema operativo UNIX se fueron esparciendo en las universidades, así como las necesidades de intercomunicarse. Por lo tanto se desarrollo el programa UUCP (UNIX to UNIX CoPy) para poder copiar archivos entre dos computadores UNIX. Además, por medio de modems y unidades de marcación telefónica automática se hizo posible poder intercambiar información entre máquinas separadas grandes distancias.

De esta forma se fueron implementando redes informalmente entre máquinas con modems y unidades de marcación automática. Sin embargo, también se tenían máquinas sin unidades de marcación automática que podían intercambiar información, ya que eran llamadas por una máquina central (con unidad de marcación automática) que recibe la información a enviar de parte de la máquina origen y luego llama a la máquina destino para enviarle dicha información.

Esta red se conoce como UUCP y tiene un control distribuido. Unas de las razones por la que es tan popular son el hecho de el gran uso de máquinas UNIX y el bajo costo de conectarse a la red, un modem y una unidad de marcación automática. Se estima que se tienen unas 10000 máquinas y un millon de usuarios. El segmento europeo de esta red se llama EUnet y otros segmentos existen en Japón, Australia y otros países. 
El servicio que brinda es el de correo electrónico, y transferencia de archivos, pero otra red muy ligada a ésta y que tiene los mismos protocolos es USENET que brinda un servicio de boletines, que pueden variar entre aspectos técnicos de lenguajes de programación a bolsas de trabajo o actividades recreacionales.

\section{CSNET Y NFSNET}

También, debido al éxito de ARPANET en la década de los años 80 se inició otra red llamada CSNET. Esta red fue creada porque la red ARPANET solo permite el acceso a universidades y particulares que tengan contratos específicos con el Departamento de Defensa de Estados Unidos. Por lo tanto, la Fundación Nacional de Ciencias de Estados Unidos (NFS) impulsó la creación de CSNET para unir los Departamentos de Ciencias de la Computación de las universidades estadounidenses.

En realidad, esta red CSNET no tiene una infraestructura propia sino que usa la infraestructura de otras redes ya establecidas. Existe una máquina central llamada CSNET RELAY en Cambridge, Massachussets.

Para usar los servicios de la red se hace por medio de ARPANET, ya que muchas universidades ya tienen acceso a ella, redes públicas X.25, PHONENET y CYPRESS. PHONENET es una red en que unos computadores se encargan de llamar a CSNET RELAY cada cierto tiempo como en el caso de UUCP, pero con un control centralizado. Finalmente, CYPRESS, es una red muy parecida a ARPANET pero a menor costo. Para ser parte de CYPRESS se necesitan IMPs (MicroVaxes), por lo cual hay que hacer una inversión extra.

El servicio que brinda CSNET es el de correo electrónico utilizando los formatos y protocolos de la red ARPANET. En el caso de utilizar PHONENET se puede además hacer transferencia de archivos y accesos remotos.

Además, la NFS decidió impulsar la creación de otra red que sirviera para accesar "super" computadoras. Por lo tanto, se necesitaba una red que pudiera manejar velocidades mayores. Esta red se llama NFSNET y sus enlaces de larga distancia transmiten a $1.5 \mathrm{Mbps}$. También hacen uso del protocolo TCP/IP.

\section{BITNET Y LA UNIVERSIDAD DE COSTA RICA}

Otra red que es muy popular en el ambiente universitario estadounidense es la red Bitnet. La diferencia de Bitnet con CSNET es que no está limitada a los Departamentos de Computación de las universidades, es decir es abierta a cualquier departamento de una universidad. Debido a que la Universidad de Costa Rica a finales de 1990 se incorporó como un nodo más de esta red se describirá su desarrollo con mayor detalle.

En 1981 cuando se hizo la primera prueba de transferencia de datos entre la Universidad de Yale y la Universidad de la Ciudad de Nueva York (CUNY) utilizando una línea telefónica rentada se podría definir como el inicio de la red Bitnet.

La idea de crear una red para compartir información entre centros de educación superior, fue impulsada por personeros de las universidades mencionadas anteriormente con base en experiencias de la red VNET; una red interna de IBM en la que se interconectaban personal técnico y administrativo de IBM en todo el mundo.

El nombre de Bitnet no se refiere a la unidad de información, bit, sino a las siglas en inglés de "porque ya es tiempo" (Because It's Time).

Aun cuando había una mayor cantidad de computadores de marca Digital que IBM en la costa Este de Estados Unidos, los computadores IBM eran más poderosos. Por lo tanto se escogió el protocolo RSCS (Remote Spooling Communications Subsystem) de la IBM como protocolo de comunicaciones.

A finales de 1981, apenas unas pocas universidades de la costa Este se habían integrado a la red. La filosofía de Bitnet era la siguiente: cada institución pagaba por su propio enlace a la red y además da facilidades para que un nuevo miembro se pudiera conectar a Bitnet a través de ella.

De esta forma se iba extendiendo la red, sin embargo, no se tuvo una conexión de costa a costa, hasta que la Universidad de California en Berkeley decidió pagar por el costo de dicho enlace con un compromiso de ayuda de otras universidades que estaban interesadas en conectarse.

A medida que iba creciendo la red se necesitaba tener un manejo formal, y una estructura central de la misma. Esto no lo podía proveer las universidades fundadoras. Por lo tanto, EDU$\mathrm{COM}$, que es una institución en la que algunos de sus miembros eran fundadores de Bitnet le pro- 
puso a IBM un proyecto para financiar un centro de soporte para Bitnet.

En 1984 IBM empez6 a financiar Bitnet y se creó un centro de información de la red, Bitnic, y un centro de desarrollo y operaciones, Bitdoc. Además IBM cedió un computador y software para el mantenimiento de la red.

En 1990 se terminó el contrato de financiamiento por parte de IBM y ahora los miembros de la red deben de financiarla. Con ese fin se formó la corporación de investigación y educación de redes CREN (Corporation for Research and Education Networking). El costo de afiliación es independiente de la utilización de la red o el número de computadores conectados y depende de la capacidad de pago de la universidad que se conecta y el costo del enlace. La afiliación está orientada a universidades pero es posible que instituciones sin fines de lucro se puedan asociar y también en algunos casos industrias de alta tecnología pero a un costo mucho mayor. Solamente se permite el intercambio de información no comercial.

Bitnet transmite a velocidades de $9600 \mathrm{bps}$, se puede comunicar con computadores IBM y a través de emuladores de RSCS con otras máquinas de diferentes sistemas operativos.

Los servicios prestados son correo electrónico (menos de 3000 líneas), transferencia de archivos (menores a 300KB), transmisión de mensajes interactivos, servicios de red (directorios, boletines, etc.), teleproceso y conexión a otras redes. De estos servicios no es muy eficiente la transmisión de mensajes interactivos y el teleproceso por la respuesta de la red, es decir se puede saturar la red. Sin embargo, existe el proyecto de Bitnet II que permitirá correr RSCS sobre el protocolo TCPIP que a su vez permite correr Telnet para aplicaciones de teleproceso.

En 1984 algunos países europeos decidieron formar una red de computadores para el intercambio de información de investigaciones entre las instituciones académicas más importantes con el nombre de EARN (Red de Investigaciones Académicas Europea) y la sede se encuentra en París.

Esta red está conectada a sus homólogas en Estados Unidos, Bitnet, y en Canadá, NetNorth. Estas tres redes forman una sola red topológicamente, aunque tengan administraciones diferentes.
Además, se puede interconectar a otras redes como ARPANET, CSNET, UUCP, NFSNET, INTERNET y SPAN (red de la Nasa).

\section{NODO UNIVERSIDAD DE COSTA RICA}

El computador utilizado como nodo en la Universidad de Costa Rica es el IBM 4381 dedicado para la investigación. El nodo se llama UCRVM2 y está conectado al controlador de comunicaciones sincrónico 3720 y además se tiene un convertidor de protocolo asincrónico 3708 para el acceso de terminales asincrónicas. Las terminales asincrónicas, ya sean internas (de la Universidad de Costa Rica) o externas (de otras instituciones del país) accesan el 3708 por medio de una PBX en la red telefónica del ICE. El computador 4381 se conecta con RACSA por medio de una línea dedicada y de ahí se conecta por medio de una línea dedicada de un satélite y un enlace de fibra óptica a la Universidad Atlántica de Florida (FAU). La velocidad del enlace es de $19.2 \mathrm{Kbps}$. En la figura No. 2 se muestra esta configuración.

Esta red es también muy popular en el ambiente de universidades, existen aproximadamente 1750 nodos en los Estados Unidos, 800 en Europa (a través de EARN), 180 en Canada (a través de NetNorth), 100 en Asia, 70 en Medio Oriente, 30 América Latina y 1 en Africa y Australia, lo cual da aproximandamente 3000 nodos en 1500 instituciones y 35 países, lo cual brinda a la Universidad de Costa Rica un excelente canal de comunicaciones con las universidades del mundo. Esto puede ayudar enormente a la capacidad de investigación de la Universidad.

\section{CONCLUSIONES}

Con base en el desarrollo que se ha descrito sobre las redes de conmutación de paquetes se puede ver que han ido aumentando su auge conforme se han rebajado los costos de los equipos de cómputo y porque se ha aumentado la cantidad de información almacenada en computadores y la necesidad de compartir esa información. Aun cuando se empezó a finales de los años 60 , el mayor auge se empieza en la década de los años 80.

También se puede observar, que aunque se habla mucho del modelo OSI, éste todavía no es totalmente implementado en la mayoría de las redes. Más bien, el protocolo de transporte más utilizado de momento es el TCP/IP y su uso en vez de disminuir va en aumento si tomamos en 
cuenta que este protocolo se está usando extensivamente en las redes de área local. También, los protocolos de aplicación de ARPANET son muy usados.

Es muy importante entonces, que se aprecie la gran oportunidad de la Universidad de Costa Rica y otras instituciones del país de poder tener acceso a una red como Bitnet. Esto abre las puertas a los profesores, investigadores y estudiantes para obtener información de lo más reciente posible de muchas universidades y el establecer y mantener contactos con otros profesores, investigadores y estudiantes en el mundo.

También es importante ver el ejemplo de otras partes del mundo donde se ha trabajado para establecer estas redes y tomarlo como guía para la Universidad de Costa Rica. Sería muy importante el establecer proyectos de investigación que ayuden a mejorar la infraestructura de comunicaciones de la Universidad, lograr un buen conocimiento de los protocolos de comunicaciones entre diferentes sistemas, como es el caso del protocolo

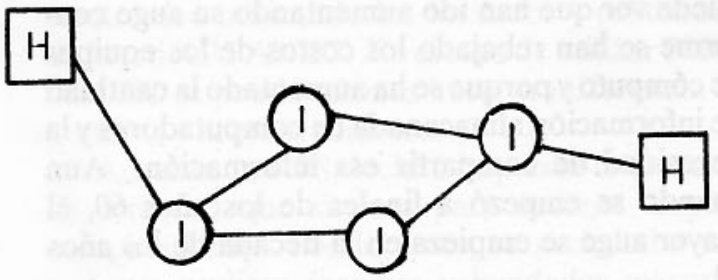

I: IMP

H: HOST
TCP/IP, y promover el desarrollo de aplicaciones que generen la necesidad de compartir información entre países de la región y del resto del mundo. De ahí, la importancia que la Universidad de Costa Rica tome el liderazgo en la investigación de redes de computadores.

\section{BIBLIOGRAFIA}

1. Tanenbaum, A.S., "Computer Networks", Prentice Hall, 1988, EUA.

2. Friend y otros, "Understanding Data Communications", Radio Shack, Primera edición, 1986, EUA.

3. Solano, E.; Navas, E.A., "Redes de Conmutación de Paquetes", Boletín Tecnico Número 26, Centro de Informática, Universidad de Costa Rica, 1988.

4. Becker, H.B., "Análisis Funcional de Redes de Información", Limusa, México, 1977.

5. Black, U., "Redes de Computadores, Protocolos, Normas e Interfaces, Macrobit, México, 1990.

6. Blanco, L.; De Teramond, G. y otros, "Red Bitnet, Guía del Usuario", Vicerrectoría de Investigación y Centro de Informática, Universidad de Costa Rica, 1990.

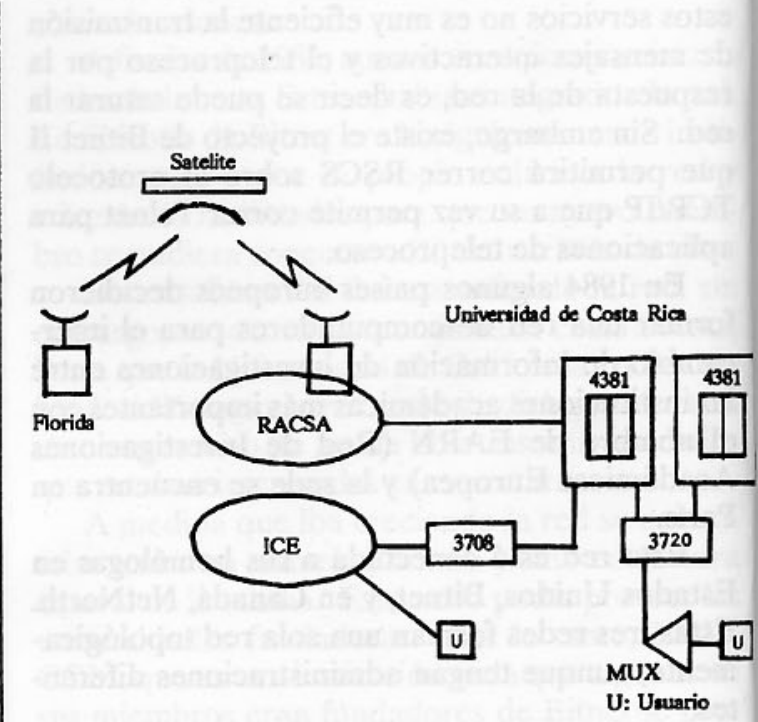

FIGURA No. 2 Conexión a BITNET 